\title{
LEGUMINOSAS CAMPESTRES DO MORRO PEDRA DO LAGARTO, SANTA MARIA, RIO GRANDE DO SUL, BRASIL ${ }^{1}$
}

\author{
HELEN FERNANDES DE MENEZES² LILIANA ESSI ${ }^{3}$
}

\section{RESUMO}

O presente trabalho teve como objetivo realizar o levantamento das espécies campestres da família Leguminosae no morro Pedra do Lagarto, distrito de Santo Antão, Santa Maria, Rio Grande do Sul, Brasil, a fim de contribuir para o conhecimento da flora da região central do estado. Tal estudo se faz necessário devido à singularidade fitogeográfica da região de Santa Maria, que se encontra em uma região de tensão ecológica entre os biomas Pampa e Mata Atlântica. Por meio de coletas quinzenais, no período de agosto de 2014 a maio de 2016, foram confirmadas 27 espécies e 17 gêneros, sendo 20 espécies pertencentes à subfamília Faboideae Rudd e sete à Mimosoideae DC. Os gêneros com maior número de espécies são Mimosa L. (seis) e Desmodium Desv. (cinco). Além da lista de espécies, este trabalho fornece dados fenológicos, fotografias e chaves analíticas para identificação dos táxons.

Palavras-chave: levantamento florístico, ecótono, campos.

\section{ABSTRACT}

[Campestrial Leguminosae from the "Pedra do Lagarto" Hill, Santa Maria, Rio Grande do Sul, Brazil].

A floristic survey of the campestrial species of Leguminosae was carried out in the "Pedra do Lagarto" Hill, Santo Antão District, Santa Maria, Rio Grande do Sul, Brazil, to increase the knowledge about the flora from the Central region of the state. Such research is necessary because of the singular phytogeographic characteristic of Santa Maria region, found on an ecological tension region between Pampa and Mata Atlântica biomes. Biweekly field collections were conducted, between August of 2014 and May of 2016, registering 27 species and 17 genera. From these species, 20 are Faboideae and seven are Mimosoideae. The genera with more species are Mimosa L. (six) and Desmodium Desv. (five). Besides the species list, this paper provides phenological data, photographs, and analytical keys for species identification.

Key words: floristic survey, ecotone, grasslands.

\section{INTRODUÇÃO}

Compreendendo, atualmente, 19.325 espécies e 727 gêneros, Leguminosae Juss. (Fabaceae Lindl.) é considerada a terceira maior família dentre as Angiospermas (Lewis et al., 2005) e apresenta ampla distribuição geográfica, ocorrendo em quase todas as regiões do mundo, especialmente em regiões tropicais e

1 Recebido em 11-09-2016 e aceito para publicação em 08-10-2016.

2 Curso de Ciências Biológicas. Universidade Federal de Santa Maria. Av. Roraima, 1000, Bairro Camobi, CEP 97105-900, Santa Maria, Rio Grande do Sul, Brasil. helmenezes@hotmail.com

3 Departamento de Biologia. Universidade Federal de Santa Maria. Av. Roraima, 1000, prédio 16, Bairro Camobi, CEP 97105-900, Santa Maria, RS, Brasil. subtropicais (Barroso et al., 1984). Muitas de suas espécies constam como o principal elemento de diferentes formações vegetais brasileiras (Lima et al., 2016). Apesar de não ser consenso entre autores, Leguminosae é considerada como uma única família, com três subfamílias: Caesalpinioideae DC., Mimosoideae DC. e Faboideae Rudd (Souza e Lorenzi, 2008), sendo consideradas monofiléticas apenas as duas últimas (Doyle e Luckow, 2003).

Esta família é reconhecida por sua importância econômica, devido ao seu potencial alimentício (amendoim, ervilha, feijão), forrageiro (ervilhacas, fava, trevos), e ornamental (Calliandra Benth., Erythrina L., Mimosa L.) (Judd, 2009). Além disso, possuem grande importância na adubação verde, pois apresentam 
associação com ectomicorrizas e com bactérias fixadoras de nitrogênio do gênero Rhizobium, capazes de converter nitrogênio atmosférico em amônia, sendo, assim, bastante utilizadas para adubação natural (Lewis et al., 2005).

De acordo com Lima et al. (2016), a diversidade de leguminosas para o Brasil é estimada em 2.803 espécies, de 222 gêneros. Destes, 15 gêneros e 1.505 espécies são consideradas endêmicas. No Rio Grande do Sul, elas compreendem 69 gêneros e 293 espécies. Estima-se que 153 espécies ocorrem em formações campestres do estado, demonstrando a importância da família na composição das formações campestres dos biomas Pampa e Mata Atlântica (Schmidt-Silveira e Miotto, 2013).

Santa Maria, município localizado na região central do Rio Grande do Sul, caracteriza-se por situar-se em região limítrofe entre os biomas Pampa e Mata Atlântica. Esse caráter fitogeográfico singular faz com que ocorram diferentes formações vegetais dentro de uma mesma região, já que os dois biomas são distintos em relação à umidade do ar, $\mathrm{pH}$ do solo e declividade (Marchiori, 2009), sendo assim considerada uma área de tensão ecológica (Cordeiro \& Hasenack, 2009) capaz de abrigar considerável biodiversidade (Cechin et al., 2009). Neste contexto, apesar do Rio Grande do Sul possuir o maior número de trabalhos taxonômicos para a família Leguminosae (Flores, 2000), o conhecimento sobre a diversidade da família ainda é insuficiente, sobretudo na região central do estado. Deste modo, levantamentos florísticos são de grande importância para o conhecimento dessas espécies.

O local escolhido para estudo foi o Morro Pedra do Lagarto, situado no distrito de Santo Antão, na região norte de Santa Maria, RS. A escolha desse local se deve à peculiaridade da formação dos morros que, segundo Sartori (2009), fazem parte do alinhamento da Serra Geral e são formados, essencialmente, por areias da Formação Botucatu e basalto proveniente de derrames de lava de origem vulcânica. Além disso, o morro estudado é composto por afloramentos rochosos circundado por formações campestres, com registros recentes de espécies raras de outras famílias botânicas (com. pess.). Desta forma, o objetivo do presente estudo foi realizar o levantamento florístico das espécies campestres da família Leguminosae do Morro Pedra do Lagarto, Distrito de Santo Antão, no município de Santa Maria.

\section{MATERIAL E MÉTODOS}

O presente estudo foi realizado no Morro Pedra do Lagarto (coordenadas 29³7’39.18"S e 53 ${ }^{\circ} 52^{\prime} 25.75^{\prime \prime O}$ ), localizado no distrito de Santo Antão, ao norte do município de Santa Maria, Rio Grande do Sul, Brasil. De acordo com a classificação climática de Köppen, o clima na região é do tipo $\mathrm{Cfa}$ (subtropical úmido), com verões quentes e sem estação seca definida. A temperatura média anual é de $19,6{ }^{\circ} \mathrm{C}$, com médias mensais de $24,8{ }^{\circ} \mathrm{C}$ (janeiro) a $14,3{ }^{\circ} \mathrm{C}$ (junho), precipitação média anual de $1.686 \mathrm{~mm}$ e chuvas bem distribuídas ao longo do ano (Moreno, 1961).

As coletas foram quinzenais, no período de agosto de 2014 a novembro de 2015, complementadas com coletas esporádicas de janeiro a maio de 2016, seguindo o método de caminhamento (com modificações), proposto por Filgueiras et al. (1994). Foram amostradas apenas espécies campestres do local. Os exemplares em fenofase reprodutiva foram coletados, bem como foram realizados registros fotográficos de alguns exemplares. A herborização e identificação foram realizadas no Laboratório de Taxonomia do Departamento de Biologia da Universidade Federal de Santa Maria. O material testemunho foi incluído no Herbário SMDB. Também foram realizadas observações acerca do ambiente e de espécies de outras famílias botânicas que ocorrem em simpatria. Para a identificação, foi utilizada literatura específica para a família, chaves analíticas, comparação com materiais de herbário e consulta a especialistas. As coletas contemplaram todas as estações do ano, tendo sido também obtidas informações sobre o ciclo de vida, dados fenológicos 
e de frutificação. A partir dos dados das coletas, das revisões no herbário SMDB e de literatura, foram elaboradas chaves para identificação dos táxons registrados.

\section{RESULTADOS E DISCUSSÃO}

A família Leguminosae está representada por 27 espécies e 17 gêneros no Morro Pedra do Lagarto (Tabela 1); algumas espécies estão representadas nas Figuras 1 e 2. A subfamilia Faboideae contribuiu com 20 espécies e 15 gêneros, sendo Desmodium Desv., com cinco espécies, o mais numeroso, seguido de Stylosanthes Sw. e Rhynchosia Lour., com duas espécies cada. Uma das espécies, Rhynchosia hauthalii (Kuntze) Grear, erva prostrada, típica de campos graminosos e arbustivos, é considerada rara para o estado do Rio Grande do Sul, por Miotto et al. (2008). Dentro desta subfamília, as tribos com maior número de espécies foram Phaseoleae Bronn ex DC., com oito, Desmodieae Hutch. (com cinco) e Dalbergieae Bronn ex DC., com quatro espécies. As tribos Crotalarieae Hutch., Genisteae Dumort. e Vicieae DC. estão representadas por uma espécie cada.

Para a subfamília Mimosoideae, o gênero Mimosa foi o mais rico em espécies, contribuindo com seis, das sete espécies encontradas desta subfamília. O gênero Desmanthus Willd. está representado pela espécie Desmanthus tatuhyensis Hoehne.

No presente estudo foi registrada a ocorrência de Mimosa sparsa Benth., um arbusto aculeado de até 3 metros de altura, e Mimosa lasiocephala Benth., pequena erva prostrada; ambas as espécies foram consideradas endêmicas para o estado Rio Grande do Sul, por Schmidt-Silveira (2015). Além disso, em seu trabalho foi verificado a existência de registros de coleta de $M$. lasiocephala apenas para a re- gião de Santa Maria, fato que poderia torná-la, ainda, uma espécie endêmica desta região.

No presente levantamento não foram registrados táxons campestres da subfamília "Caesalpinioideae" que são comuns em levantamentos florísticos de Leguminosae, tais como espécies do gênero Chamaecrista (Camargo \& Miotto, 2004, Dambros et al., 2004). Espécies arbustivas e arbóreas da referida subfamília foram encontradas em áreas de mata, na encosta do morro; tais áreas, entretanto, não foram contempladas no presente estudo.

Os resultados evidenciam que a área investigada apresenta uma importante riqueza em leguminosas da subfamília Faboideae, uma vez que o número de espécies encontradas assemelha-se ao registrado em ecossistemas campestres de outros locais, tais como o verificado por Dambros et al. (2004) no campus Santa Maria, da Universidade Federal de Santa Maria (30 espécies de Faboideae, pertencentes a 22 gêneros), e por Silva et al. (2013) no campus Palmeira das Missões, da mesma universidade, onde foram encontradas 22 espécies, de 14 gêneros de Faboideae. Por outro lado, a maioria das espécies encontradas de Mimosoideae não figuram em outros levantamentos da região, nem mesmo para o estado do Rio Grande do Sul, conforme destacado anteriormente.

No presente estudo não foram registradas espécies exóticas de Leguminosae que são comuns em levantamentos florísticos, tais como espécies do gênero Medicago L. e Trifolium L. Porém, há registro de espécies exóticas de outras famílias botânicas, casos de Melinis repens (Willd.) Zizka (Poaceae) e Pinus taeda L. (Pinaceae). Apesar disso, de modo geral, a área ainda é relativamente conservada e abriga diversas espécies raras de outras famílias, tais como Cactaceae, Bromeliaceae, e Asteraceae (com. pess.). 


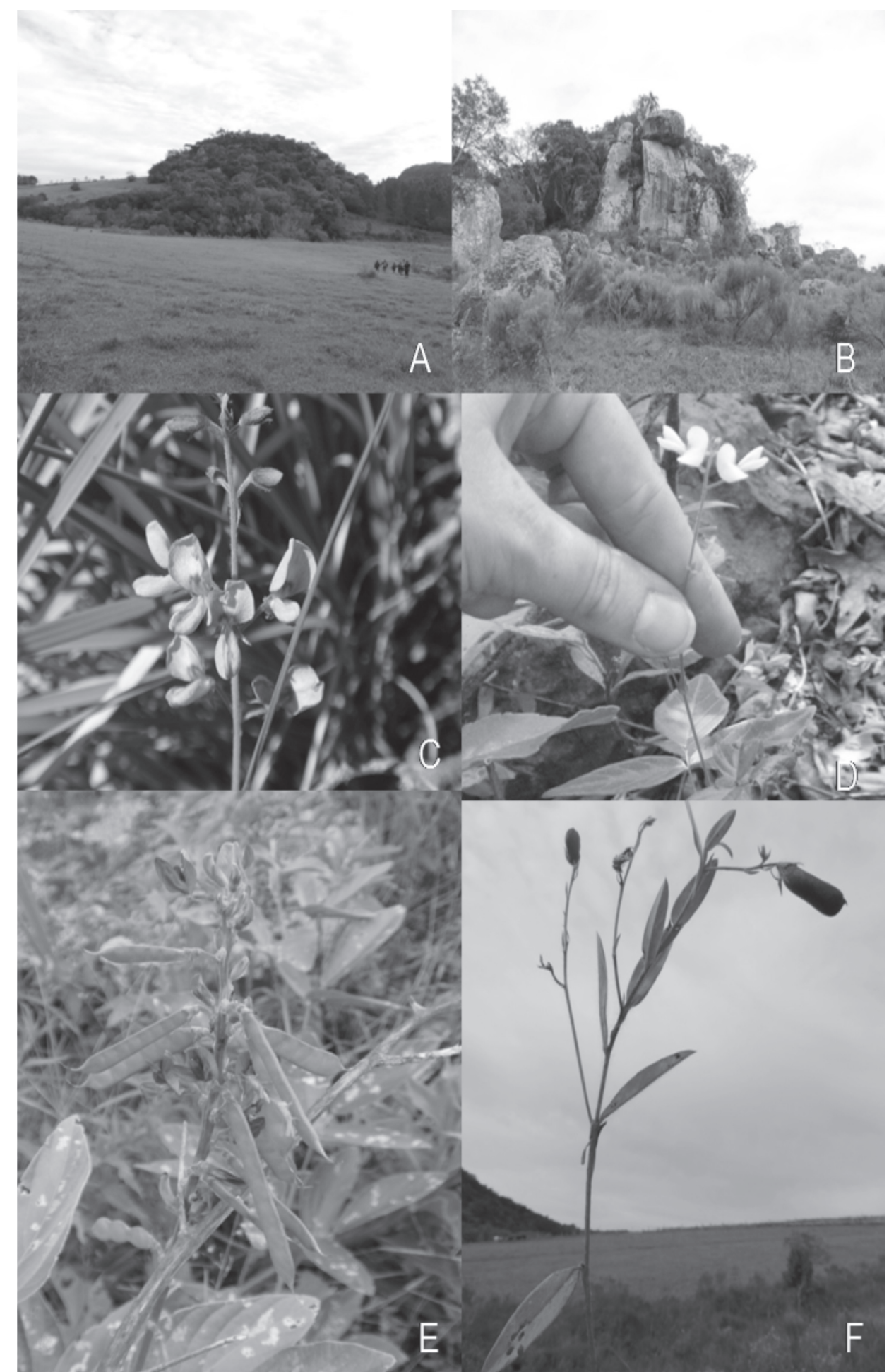

FIGURA 1. A - Detalhe de afloramentos rochosos no morro. B - Vista geral do morro. C - Desmodium incanum. D - Desmodium affine. E-Calopogonium caeruleum. F-Crotalaria tweediana 


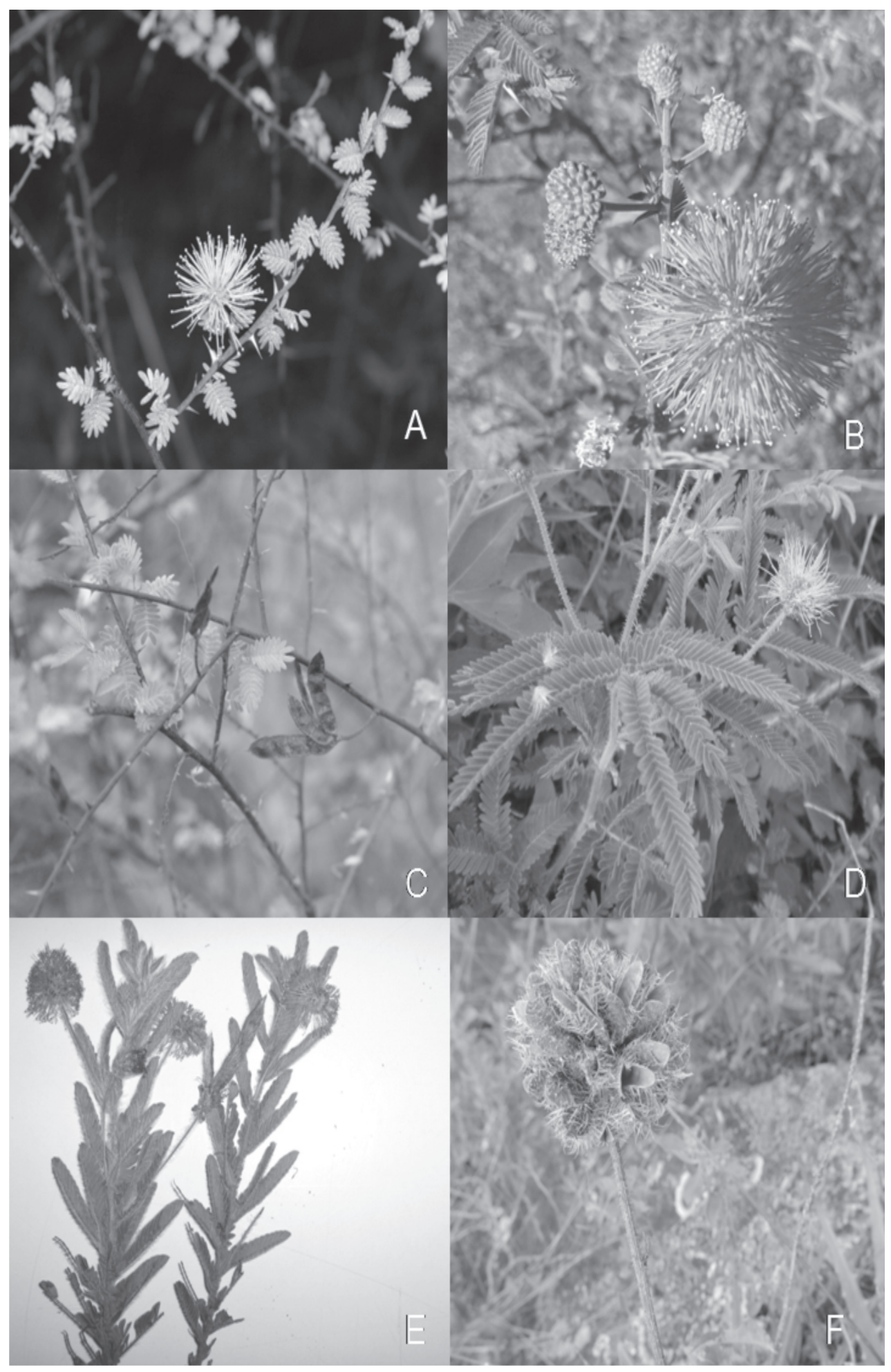

FIGURA 2. Espécies de Mimosa. A - Detalhe dos acúleos e folhas de Mimosa sparsa. B - Detalhe da inflorescência de M. sparsa. C - Detalhe dos frutos de M. sparsa. D - Mimosa lasiocephala. E - Exsicata de M. lasiocephala, com frutos. F - Frutos de Mimosa dolens subsp. rigida. 
FIGURA 1 - Lista de espécies de Leguminosae do morro Pedra do Lagarto, com informações de fenologia (floração e frutificação), distribuídas conforme as tribos.

\begin{tabular}{|c|c|c|}
\hline Espécie & Fenologia & Material selecionado \\
\hline \multicolumn{3}{|l|}{ Subfamília Faboideae } \\
\hline \multicolumn{3}{|l|}{ Tribo Crotalarieae } \\
\hline Crotalaria tweediana Benth. & março a outubro & H.F. de Menezes 076 (SMDB) \\
\hline \multicolumn{3}{|l|}{ Tribo Dalbergieae } \\
\hline Aeschynomene falcata (Poir.) DC. & novembro a março & H.F. de Menezes 044 (SMDB) \\
\hline Stylosanthes leiocarpa Vogel & dezembro a abril & H.F. de Menezes 040 (SMDB) \\
\hline Stylosanthes montevidensis Vogel & novembro a março & H.F. de Menezes 070 (SMDB) \\
\hline Zornia reticulata $\mathrm{Sm}$. & $\begin{array}{l}\text { dezembro a abril (TANNUS et al. } \\
\text { 2006) }\end{array}$ & H.F. de Menezes 068 (SMDB) \\
\hline \multicolumn{3}{|l|}{ Tribo Desmodieae } \\
\hline Desmodium adscendens (Sw.) DC. & setembro a abril & H.F. de Menezes 043 (SMDB) \\
\hline Desmodium affine Schltdl. & novembro & H.F. de Menezes 018 (SMDB) \\
\hline Desmodium barbatum (L.) Benth. & dezembro a abril & H.F. de Menezes 064 (SMDB) \\
\hline Desmodium cuneatum Hook. \& Arn. & setembro a janeiro & H.F. de Menezes 062 (SMDB) \\
\hline Desmodium incanum (Sw.) DC. & agosto a abril & H.F. de Menezes 017 (SMDB) \\
\hline \multicolumn{3}{|l|}{ Tribo Genisteae } \\
\hline Lupinus bracteolaris Desr. & $\begin{array}{l}\text { agosto a janeiro (SCHMIDT- } \\
\text { SILVEIRA E MIOTTO 2013) }\end{array}$ & H.F. de Menezes 002 (SMDB) \\
\hline \multicolumn{3}{|l|}{ Tribo Phaseoleae } \\
\hline Calopogonium caeruleum (Benth.) C. Wright & novembro a janeiro & H.F. de Menezes 063 (SMDB) \\
\hline Collaea stenophylla (Hook. \& Arn.) Benth. & janeiro & H.F. de Menezes 037 (SMDB) \\
\hline Eriosema tacuaremboense Arechav & setembro a novembro & H.F. de Menezes 012 (SMDB) \\
\hline Galactia neesii DC. & outubro & H.F. de Menezes 008 (SMDB) \\
\hline Macroptilium erythroloma (Mart. ex Benth.) Urb. & outubro a abril & H.F. de Menezes 009(SMDB) \\
\hline Rhynchosia corylifolia Mart. ex Benth. & outubro a janeiro & H.F. de Menezes 013 (SMDB) \\
\hline Rhynchosia hauthalii (Kuntze) Grear & novembro & H.F. de Menezes 021 (SMDB) \\
\hline Vigna peduncularis Fawc. \& Rendle & dezembro a março & H.F. de Menezes 004 (SMDB) \\
\hline \multicolumn{3}{|l|}{ Subfamília Mimosoideae } \\
\hline \multicolumn{3}{|l|}{ Tribo Mimoseae } \\
\hline Desmanthus tatuhyensis Hoehne & outubro a dezembro & H.F. de Menezes 052 (SMDB) \\
\hline Mimosa cruenta Benth. & julho a outubro & H.F. de Menezes 054 (SMDB) \\
\hline Mimosa dolens subsp. rigida (Benth.) Barneby & junho a janeiro & H.F. de Menezes 038 (SMDB) \\
\hline Mimosa dutrae Malme & outubro a janeiro & H.F. de Menezes 005 (SMDB) \\
\hline Mimosa lasiocephala Benth. & outubro a janeiro & H.F. de Menezes 065 (SMDB) \\
\hline Mimosa rupestris Benth. & agosto & H.F. de Menezes 001 (SMDB) \\
\hline Mimosa sparsa Benth. & $\mathrm{o}$ ano inteiro & H.F. de Menezes 073 (SMDB) \\
\hline
\end{tabular}

\section{Chave 1. Chave para identificação das subfamílias de Leguminosae do Morro Pedra do Lagarto.}

1. Flores actinomorfas. Cálice e corola reduzidos. Estames 4 a numerosos.

Folhas bipinadas Mimosoideae

2. Flores zigomorfas. Cálice e corola não reduzidos e bem diferenciados.

10 estames. Folhas nunca bipinadas Faboideae 


\section{Chave 2. Chave para identificação das espécies da subfamília Faboideae no morro Pedra do Lagarto.}

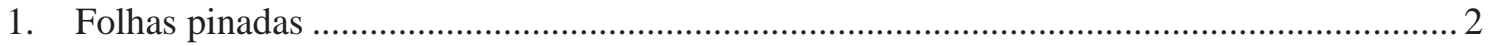

1'. Folhas unifolioladas, bifolioladas ou trifolioladas ............................................................... 3

2. Flores de cor amarela. Folhas sem gavinhas.

Brácteas presentes

Aeschynomene falcata

2'. Flores de cor rosa a lilás. Folhas com gavinhas.

Brácteas ausentes

Vicia graminea

3. Folhas bifolioladas. Flores protegidas por brácteas foliáceas Zornia reticulata

3'. Folhas unifolioladas ou trifolioladas. Flores não protegidas por rácteas foliáceas

4. Folhas unifolioladas, elípticas a obovadas. Legume inflado

Crotalaria tweediana

4'. Folhas trifolioladas. Legume não inflado

5. Ervas volúveis. Flores cor salmão. Folíolos panduriformes ......... Macroptilium erythroloma

5'. Ervas não-volúveis, eretas ou prostradas. Flores com variadas cores, exceto salmão. Folíolos não panduriformes.

6. Flores de cor lilás. Estandarte com mancha central branca. Racemos terminais

6'. Flores de cor branca, amarela ou lilás. Estandarte sem mancha central branca.

Racemos terminais a axilares .................................................................................. 7

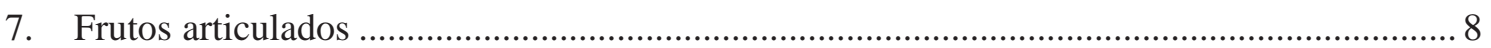

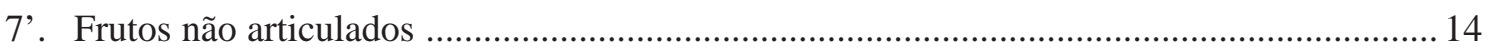

8. Flores amarelas. Lomento com 1 ou dois artículos férteis ............................................... 9

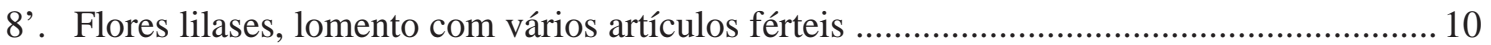

9. Folíolos linear-lanceolados. Folhas caducas após frutificação. Lomentos com um artículo fértil ..................................................................... Stylosanthes montevidensis

9’. Folíolos elípticos. Folhas persistentes após frutificação. Lomento com dois artículos férteis Stylosanthes leiocarpa

10. Eixo da inflorescência com até $5 \mathrm{~cm}$ de comprimento Desmodium barbatum

10'. Eixo da inflorescência maior que $5 \mathrm{~cm}$ de comprimento 11

11. Estípulas ovadas. Flores com coloração branca a rosa clara. Lomento com mancha linear amarela na margem inferior

11'. Estípulas triangulares a estreito triangulares. Flores com coloração lilás, roxa, violeta ou rosa. Lomento sem mancha linear amarela na margem inferior Desmodium affine

12'. Estípulas livres entre si. Plantas não estoloníferas

Desmodium incanum

13. Folhas subsésseis. Folíolos elípticos a estreito elípticos. Estípulas estreito-triangulares. Presença de xilopódio Desmodium cuneatum

13'. Folhas pecioladas. Folíolos obovados a orbiculares. Estípulas triangulares. Ausência de xilopódio Desmodium adscendens

14. Legumes com duas sementes. Folíolos com glândulas punctiformes

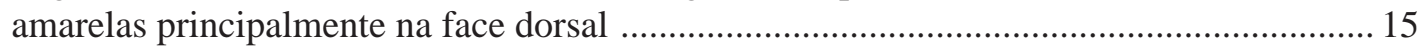

14'. Legumes com mais de duas sementes. Folíolos semglândulas punctiformes ...................... 17

15. Ervas ou subarbustos eretos. Folíolos elípticos a estreito elípticos. Plantas com pilosidade branca-prateada Eriosema tacuaremboense 15'. Ervas prostradas ascendentes a eretas. Folíolos obovados, largo-ovalados a orbiculares. 
Plantas sem pilosidade branca-prateada

16. Folhas basais sempre trifolioladas. Folíolos não reticulado-rugosos (bulados).

Estípulas cordado-ovaladas Rhynchosia corylifolia

16'. Folhas basais as vezes unifolioladas. Folíolos reticulado-rugosos em ambas as faces. Estípulas lanceoladas a triangulares Rhynchosia hauthalii

17. Folhas pinadas trifolioladas. Flores azuis. Racemos terminais e axilares Calopogonium caeruleum

17'. Folhas digitado trifolioladas. Flores brancas a lilás. Racemos axilares . 18

18. Flores brancas. Estandarte flabeliforme. Cálice com cinco lacínias. Folhas sésseis Collaea stenophylla

18’. Flores lilases. Estandarte não flabeliforme. Cálice com quatro lacínias. Folhas pecioladas Galactia neesii

\section{Chave 3. Chave para as espécies da subfamília Mimosoideae do morro Pedra do Lagarto}

1. Androceu com 8 a muitos estames. Folhas com 2 a muitos pares de folíolos ...................... 2

1'. Androceu com 4 estames. Folhas com 1 par de folíolos .................................................... 3

2. Androceu com 8 estames. Estames róseos a lilás. 2-3 pares de folíolos. Ramos com tricomas glandulares. Ausência de xilopódio ............. Mimosa cruenta (Secção Batocaulon)

2'. Androceu com 10 ou mais estames. Estames brancos. 2-5 pares de folíolos. Ramos sem tricomas glandulares. Presença de xilopódio Desmanthus tatuhyensis

3. Plantas armadas Mimosa sparsa

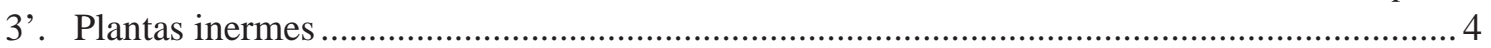

4. Folhas pequenas (10-25 mm). Pecíolos muito curtos $(1-3 \mathrm{~mm}$............................................ 5

4'. Folhas grandes (30-80 mm). Pecíolos compridos (10-85 mm ............................................... 6

5. Folíolos 2-4 mm. Pecíolos 1-2 mm. 25 a muitos pares de foliólulos. Ramos com indumento estrigoso. Fruto não- articulado Mimosa rupestris

5'. Folíolos 1-3 mm. Pecíolos sésseis. 10-19 pares de foliólulos. Ramos com indumento lanuginoso. Fruto articulado Mimosa lasiocephala

6. Ervas eretas. Inflorescência capituliforme. Folíolos 22-30 mm. 5-9 pares de foliólulos. Margem dos foliólulos com tricomas ganchosos.

Frutos agrupados uniarticulados formando uma cabeça Mimosa dolens subsp. rigida

6.' Ervas prostradas estoloníferas. Inflorescência espiciforme. Folíolos 6-11 mm, 9 a muitos pares de foliólulos. Margem dos foliólulos lisa. Frutos com 1-3 artículos não formando uma cabeça Mimosa dutrae

\section{AGRADECIMENTO}

À Fernanda Schmidt Silveira, pelo auxílio na identificação do gênero Mimosa. Às servidoras do SMDB, Benardete Panno e Tânia Boucinha, pela solicitude prestada durante as consultas ao acervo.

\section{REFERÊNCIAS BIBLIOGRÁFICAS}

BARROSO, G. M.; PEIXOTO, A. L.; COSTA, C. G.; LIMA, H. C.; ICHASO, C. L. F.; GUIMARÃES, E. F. Sistemática de Angiospermas do
Brasil. Viçosa, MG: Imprensa Universitária da Universidade Federal de Viçosa, 1984. 377p.

CAMARGO, R. A.; MIOTTO, S. T. S. O gênero Chamaecrista Moench (LeguminosaeCaesalpinioideae) no Rio Grande do Sul. Iheringia, Série Botânica, v. 59, n. 2, p. 131148, 2004.

CECHIN, S. Z.; MORAIS, A. B. B.; CÁCERES, N. C.; SANTOS, S.; KOTZIAN, C. B.; BEHR, E. R.; ARRUDA, J. F.; DELLA-FLORA, F. A fauna de Santa Maria. Ciência \& Ambiente, Santa Maria, v.1, n.38, p. 113-144, 2009. 
CORDEIRO, J. L. P.; HASENACK, H. Cobertura vegetal atual do Rio Grande do Sul. In: PILLAR, V. P. et al. Campos Sulinos: Conservação e uso sustentável da biodiversidade. Brasília: MMA, 2009. p. 285-299.

DAMBROS, V. S.; EISINGER, S. M.; CANTO DOROW, T. S. Leguminosae do Campus da Universidade Federal de Santa Maria, Santa Maria, RS, Brasil. Ciência e Natura, Santa Maria, v. 26, n. 2, p. 43, 2004.

DOYLE, J. J.; LUCKOW, M. A. The rest of the iceberg. Legume diversity and evolution in a phylogenetic context. Plant Physiology, v. 131, n. 3, p. 900-910, 2003.

FILGUEIRAS, T. S. et al. Caminhamento: um método expedito para levantamentos florísticos qualitativos. Cadernos de Geociências, v. 12, n. 1, p. 39-43, 1994.

FLORES, A. S. O gênero Crotalaria L. (LeguminosaeFaboideae) na Região Sul do Brasil. Dissertação (Mestrado em Botânica) - Universidade Federal do Rio Grande do Sul, Porto Alegre, 2000.

JUDD, W. S.; CAMPBELL, C. S.; KELLOGG, E. A.; STEVENS, P. F.; DONOGHUE, M. J. Sistemática vegetal: um enfoque filogenético. Porto Alegre: Artmed, 2009. 632p.

LEWIS, G.; SCHRIRE, B.; MACKINDER, B.; LOCK, M. Legumes of the world. Kew: Royal Botanic Gardens, 2005. 577p.

LIMA, H. C.; QUEIROZ, L. P.; MORIM, M. P.; SOUZA, V. C.; DUTRA, V. F.; BORTOLUZZI, R. L. C.; IGANCI, J. R. V.; FORTUNATO, R. H.; VAZ, A. M. S. F.; SOUZA, E. R.; FILARDI, F. L. R.; VALLS, J. F. M.; GARCIA, F. C. P.; FERNANDES, J. M.; MARTINS-DA-SILVA, R. C. V.; PEREZ, A. P. F.; MANSANO, V. F.; MiOTTO, S. T. S.; TOZZI, A. M. G. A.; MEIRELES, J. E.; LIMA, L. C. P.; OLIVEIRA, M. L. A. A.; FLORES, A. S.; TORKE, B. M.; PINTO, R. B.; LEWIS, G. P.; BARROS, M. J. F.; SCHÜTZ, R.; PENNINGTON, T.; KLITGAARD, B. B.; RANDO, J. G.; SCALON, V. R.; CARDOSO, D. B. O. S.; COSTA, L. C.; SILVA, M. J.; MOURA, T. M.; BARROS, L. A. V.; SILVA, M. C. R.; QUEIROZ, R. T.; SARTORI, A. L. B.; CAMARGO, R. A.; LIMA, I. B.; COSTA, J.; SOARES, M. V. B.; SNAK,
C.; SÃO-MATEUS, W.; FALCÃO, M. J.; MARTINS, M. V.; REIS, I. P.; CORDULA, E.; MONTEIRO, V. 2016. Fabaceae. In: Lista de Espécies da Flora do Brasil. Jardim Botânico do Rio de Janeiro. Disponivel em: http: // floradobrasil.jbrj.gov.br/jabot/floradobrasil/ FB115. Acesso em: 01 Jun. 2016.

MARCHIORI, J. N. C. A Vegetação em Santa Maria. In: História natural de Santa Maria. Ciência e Ambiente, Santa Maria, v.1, n.38, p. 93-112, 2009.

MIOTTO, S. T. S.; LÜDTKE, R.; OLIVEIRA, M. L. A. A. A família Leguminosae no Parque Estadual de Itapuã, Viamão, Rio Grande do Sul, Brasil. Revista Brasileira de Biociências, v. 6, n. 3, 2008 .

MORENO, J. A. Clima do Rio grande do Sul. Boletim Geográfico do Rio Grande do Sul, n. 11, p. 4983, 1961.

SARTORI, P. L. P. Geologia e geomorfologia de Santa Maria. In: História natural de Santa Maria. Ciência e Ambiente, Santa Maria, v.1, n. 38, p. 19-42, 2009.

SCHMIDT-SILVEIRA, F; MIOTTO, S. T. S. A família Fabaceae no Morro Santana, Porto Alegre, Rio Grande do Sul, Brasil: aspectos taxonômicos e ecológicos. Revista Brasileira de Biociências, v. 11, n. 1, 2013.

SCHMIDT-SILVEIRA, F. Estudos taxonômicos em Mimosa L. seção Mimosa (Fabaceae, Mimosoideae) no Rio Grande do Sul. Dissertação de Mestrado. Programa de Pós-Graduação em Botânica, UFRGS, 2015. 260 p.

SILVA, L. N.; LOCATELLI, A. P. C.; ESSI, L. Leguminosas campestres da Universidade Federal de Santa Maria, campus Palmeira da Missões, RS, Brasil. Revista Brasileira de Biociências, v. 11, n. 2, 2013.

SOUZA, V. C.; LORENZI, H. Botânica sistemática: guia ilustrado para identificação das famílias de fanerógamas nativas e exóticas no Brasil, baseado em APG II. 2. ed. Nova Odessa, SP: Instituto Plantarum, 2008. 704 p.

TANNUS, J. L. S.; ASSIS, M. A.; MORELLATO, L. P. C. Fenologia reprodutiva em campo sujo e campo úmido numa área de cerrado no sudeste do Brasil, Itirapina-SP. Biota Neotropica, v. 6 , n. 3, p. 1-27, 2006. 